Homology, Homotopy and Applications, vol.10(1), 2008, pp.29-39

\title{
EXCISION FOR $K$-THEORY OF CONNECTIVE RING SPECTRA
}

\author{
BJØRN IAN DUNDAS AND HARALD ØYEN KITTANG
}

(communicated by Charles Weibel)

\begin{abstract}
We extend Geisser and Hesselholt's result on "bi-relative $K$ theory" from discrete rings to connective ring spectra. That is, if $\mathcal{A}$ is a homotopy cartesian $n$-cube of ring spectra (satisfying connectivity hypotheses), then the $(n+1)$-cube induced by the cyclotomic trace
\end{abstract}

$$
K(\mathcal{A}) \rightarrow T C(\mathcal{A})
$$

is homotopy cartesian after profinite completion. In other words, the fiber of the profinitely completed cyclotomic trace satisfies excision.

\section{Introduction}

Topological $K$-theory is a cohomology theory. Most importantly it satisfies excision; so if for instance $X$ is a CW-complex defined by gluing two subcomplexes $X^{1}$ and $X^{2}$ along their intersection $X^{12}$, then the Mayer-Vietoris sequence

$$
\cdots \rightarrow K^{0}(X) \rightarrow K^{0}\left(X^{1}\right) \oplus K^{0}\left(X^{2}\right) \rightarrow K^{0}\left(X^{12}\right) \rightarrow K^{1}(X) \rightarrow \cdots
$$

is exact. In other words, the square of spectra

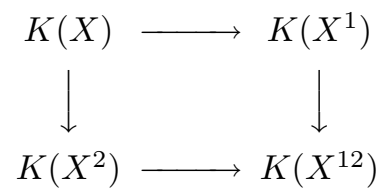

is homotopy cartesian.

This is not true in algebraic $K$-theory: given maps $f^{2}: A^{2} \rightarrow A^{12}$ and $f^{1}: A^{1} \rightarrow A^{12}$ of rings, let $A^{0}=A^{1} \times{ }_{A^{12}} A^{2}$ be the pullback (corresponding in the commutative case to $\operatorname{Spec}\left(A^{0}\right)$ being formed by gluing $\operatorname{Spec}\left(A^{1}\right)$ and $\operatorname{Spec}\left(A^{2}\right)$ along $\left.\operatorname{Spec}\left(A^{12}\right)\right)$. Then

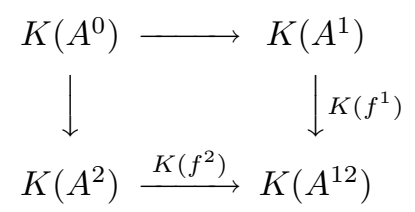

need not be homotopy cartesian. It is true that under surjectivity conditions on $f^{1}$

Received May 1, 2007, revised October 26, 2007; published on November 29, 2007.

2000 Mathematics Subject Classification: 19D55, 55P43, 18G30, 19 C40.

Key words and phrases: algebraic $K$-theory, excision, ring spectrum.

This article is available at http://intlpress.com/HHA/v10/n1/a2

Copyright (C) 2007, International Press. Permission to copy for private use granted. 
and $f^{2}$ the Mayer-Vietoris sequence is exact in low degrees, but this does not continue in higher degrees. See [19] for an amusing account, for instance showing that even if all maps in the square are surjective, there does not exist a functor $K_{3}$ such that the Mayer-Vietoris sequence can be extended.

In a series of papers $([\mathbf{1 1}],[\mathbf{8}],[\mathbf{1 0}],[\mathbf{9}])$ Geller, Reid and Weibel explored the idea that cyclic homology should be a precise measure for the failure of excision in the algebraic $K$-theory of $\mathbf{Q}$-algebras, and did some conjectural calculations. The problem remained open (although it IS an exercise in [13]), until Cortiñas released a preprint [3] claiming the conjecture using Suslin and Wodzicki's results on nonunital rings $[\mathbf{1 8}]$.

In a recent preprint [7] Geisser and Hesselholt give the corresponding result after profinite completion, with the difference that cyclic homology has to be replaced by topological cyclic homology $T C$. The result from [7] we generalize is the following. Let

$$
\mathcal{A}=\left\{\begin{array}{ccc}
A^{0} & & A^{1} \\
\downarrow & & \downarrow^{1} \\
A^{2} & & A^{12}
\end{array}\right\}
$$

be a cartesian square of discrete rings with $f^{1}$ surjective; then the cube $K(\mathcal{A}) \rightarrow$ $T C(\mathcal{A})$ is homotopy cartesian after profinite completion. A word of explanation: $K(\mathcal{A})$ is the square of spectra

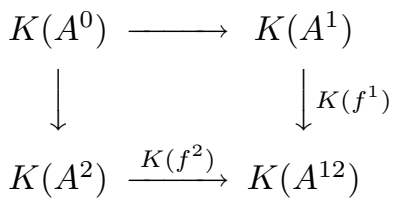

and similarly for $T C(\mathcal{A})$. The cyclotomic trace $K \rightarrow T C$ then gives a map of squares. Considering the map of squares as a cube, the theorem states that this cube is homotopy cartesian after profinite completion.

Another appealing formulation is that the homotopy fiber of the profinitely completed cyclotomic trace satisfies excision.

In this paper we extend the theorem from rings to ring spectra: let $\mathbf{S}$ be the sphere spectrum in any of the popular theories of spectra with strict symmetric monoidal smash product; then we have the following result.

Theorem 1.1. Consider a homotopy cartesian diagram $\mathcal{A}$ of connective $\mathbf{S}$-algebras

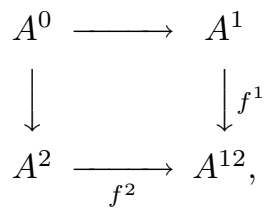

where $f^{1}$ is 0-connected. Then the cube

$$
K(\mathcal{A}) \longrightarrow T C(\mathcal{A})
$$

induced by the cyclotomic trace, is homotopy cartesian after profinite completion. 
The proof of the theorem is delightfully simple. It follows by the theorems of McCarthy [16], the first author [4], and Geisser and Hesselholt [7] in addition to an elementary observation about homotopy cartesian diagrams of ring spectra.

Actually we prove a stronger and more technical result in Proposition 2.1 and then show that the conditions in Theorem 1.1 imply those of Proposition 2.1.

We mention that Theorem 1.1 holds integrally by work in progress of the second author.

Example 1.2. As an example, let $k$ be a connective $\mathbf{S}$-algebra, and consider the "cusp" $C$ over $k$ gotten by the homotopy pullback

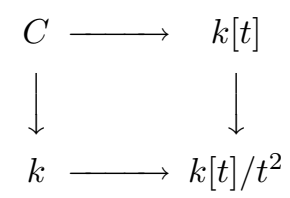

(if $k$ is a discrete ring, $C \cong k[x, y] /\left(x^{2}-y^{3}\right)$, hence the name). Letting $F$ be the profinite completion of the homotopy fiber of the cyclotomic trace, the diagram remains homotopy cartesian after applying $F$. However, by [4] the map $F(k) \rightarrow F\left(k[t] / t^{2}\right)$ is an equivalence, and so $F(C) \rightarrow F(k[t])$ is an equivalence. The rightmost term may then be calculated from $N i l$-terms (if $k$ is not "regular", then the Nil-term in $K(k[t]) \simeq K(k) \times N i l_{k}^{K}$ will not vanish) and $T C(k[t])$.

Hence, one can calculate $K(C)$ if one can describe $T C(C), T C(k[t]), K(k)$ and the Nil-terms (and all the maps connecting them).

Remark 1.3. One might be tempted to believe that the crucial condition on our square of $\mathbf{S}$-algebras is that it is homotopy cartesian, but unfortunately the conclusion of the theorem is false without the surjectivity assumption on $\pi_{0} f^{1}$. A counterexample can be derived without calculations from the homotopy cartesian square (the maps are the natural inclusions)

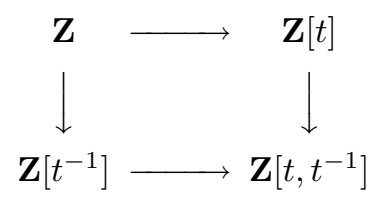

and its sibling with the $p$-adic integers $\mathbf{Z}_{p}$ instead of $\mathbf{Z}$. By the fundamental theorem of algebraic $K$-theory, the iterated fibers of the two $K$-theory squares are $K(\mathbf{Z})$ and $K\left(\mathbf{Z}_{p}\right)$ respectively. They are very different: $K_{1}(\mathbf{Z}) \cong \mathbf{Z} / 2$ and $K_{1}\left(\mathbf{Z}_{p}\right) \cong \mathbf{Z} /(p-1) \times$ $\mathbf{Z}_{p}$. On the other hand, the topological cyclic homology of the integral and $p$-adic square agree after $p$-completion.

The example above has the deficiency that Milnor's theorem [1, Section IX.5] does not apply: the associated square of categories of finitely generated projective modules is not a fiber square. We know of no examples of squares of rings for which the Milnor theorem applies where the conclusion of the main theorem does not hold.

A natural conjecture would be that the fiber of the cyclotomic trace takes fiber squares of exact categories to homotopy cartesian squares. Beyond the obvious extensions that follow from the theorems of Cortiñas and Geisser-Hesselholt, the case of the projective line is our only support for this conjecture. 
There is a direct proof of the extension of Geisser and Hesselholt's theorem to simplicial rings not using Goodwillie's conjecture [16], [4]. This proof is interesting in that it gives a hands on approach to the problem, and conceivably a way to weaken the conditions of the theorem. We will not pursue those questions here.

\subsection{Plan}

In Section 2 we prove a proposition that turns out to be stronger than the main theorem 1.1. We do not require the square of $\mathbf{S}$-algebras to be homotopy cartesian, but rather impose criteria on the path components.

In Section 3 we address the problem that $\pi_{0}$ does not send homotopy cartesian squares to cartesian squares. We also prove some multirelative extensions.

\subsection{Conventions}

The algebraic $K$-theory discussed in this paper is the nonconnective version of algebraic $K$-theory as defined by Thomason [20, Section 6] extended to connective S-algebras. Thomason's construction is functorial, and is also performed on the cyclotomic trace (see below). Since for a connective $\mathbf{S}$-algebra $A$ we have that $K_{1}(A) \cong K_{1}\left(\pi_{0} A\right)$, we get little new: $K_{i}(A) \cong K_{i}\left(\pi_{0} A\right)$ for all $i \leqslant 1$. In particular, if $A \rightarrow B$ induces an isomorphism on $\pi_{0}$ (as is the case for the vertical maps in the theorem), then the relative groups nonconnective $K$-theory vanish in negative dimensions. The same considerations apply to $T C$.

Topological cyclic homology $T C$ is taken to be integral topological cyclic homology as defined by Goodwillie [12], but appears in this paper only after profinite completion, and so agrees with the product over all primes $p$ of the $p$-completion of the $p$-typical version $T C(-; p)$ appearing in [2]. The cyclotomic trace is given as in [5].

All displayed diagrams commute. The term "connective" is reserved for -1-connected spectra.

\section{Sufficient conditions on the path components}

In this section we prove Proposition 2.1 (and a multirelative version, Corollary 2.2) that turns out to be stronger than the main theorem 1.1. We do not require the square of S-algebras to be homotopy cartesian, but rather impose criteria on the path components.

Proposition 2.1. Let $\mathcal{A}$ be a diagram

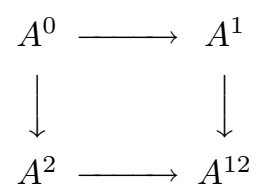

of connective $\mathbf{S}$-algebras such that $\pi_{0} A^{1} \rightarrow \pi_{0} A^{12}$ is surjective and the induced map of rings

$$
\pi_{0} A^{0} \rightarrow \pi_{0} A^{1} \times_{\pi_{0} A^{12}} \pi_{0} A^{2}
$$

is a surjection with nilpotent kernel. Then the cube

$$
K(\mathcal{A}) \rightarrow T C(\mathcal{A})
$$


induced by the trace map is homotopy cartesian after profinite completion.

Proof. Let $F$ be the profinite completion of the homotopy fiber of the cyclotomic trace $K \rightarrow T C$. Since $\pi_{0} A^{1} \rightarrow \pi_{0} A^{12}$ is surjective, Geisser and Hesselholt's theorem implies that the square

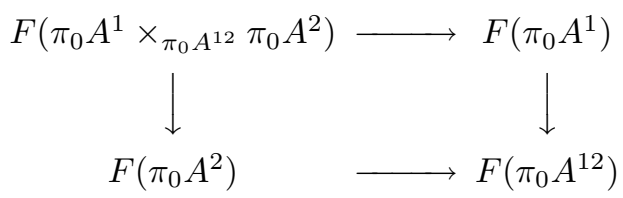

is homotopy cartesian. The assumption that $\pi_{0} A^{0} \rightarrow \pi_{0} A^{1} \times_{\pi_{0}} A^{12} \pi_{0} A^{2}$ is a surjection with nilpotent kernel, opens for the use of McCarthy's theorem [16] and we may conclude that

$$
F\left(\pi_{0} A^{0}\right) \rightarrow F\left(\pi_{0} A^{1} \times_{\pi_{0}} A^{12} \pi_{0} A^{2}\right)
$$

is an equivalence. Hence the square $F\left(\pi_{0} \mathcal{A}\right)$ is homotopy cartesian.

Now, by [4], each of the vertical maps in the cube

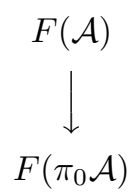

are equivalences, and the result follows.

The above results automatically give theorems about $n$-cubes for $n \geqslant 1$. Recall that if $S$ is a finite set, then an $S$-cube is a functor from the category $\mathcal{P} S$ of subsets of $S$, and that if $|S|$ is the cardinality of $S$, one often uses the term $|S|$-cube. Hence a 0 -cube is an object, a 1-cube is a map and a 2-cube is a commuting square.

Corollary 2.2. Let $\mathcal{A}$ be an $S$-cube of connective $\mathbf{S}$-algebras such that for all $U \subseteq S$ the canonical map

$$
p^{U}: \pi_{0} A^{U} \rightarrow \underset{U \subsetneq T \subseteq S}{\lim } \pi_{0} A^{T}
$$

is surjective, and in addition that $p^{\emptyset}$ has nilpotent kernel. Then the $(|S|+1)$-cube

$$
K(\mathcal{A}) \rightarrow T C(\mathcal{A})
$$

induced by the cyclotomic trace is homotopy cartesian after profinite completion.

Proof. Note that the surjectivity condition on the cube is symmetric in the sense that the condition is satisfied for all subcubes. In particular, all maps in the cube are 0-connected. By the same reasoning as in Proposition 2.1 we may immediately reduce to the case of discrete rings. For concreteness, let $S=\{1, \ldots, n\}$, and assume by induction that the corollary has been proven for cubes of cardinality less than $n$.

Write $S^{\prime}$ for $\{1, \ldots, n-1\}$. Let $\mathcal{A}[\emptyset]$ be the cartesian $(n-1)$-cube obtained by restricting the functor $\mathcal{A}$ to $\mathcal{P} S^{\prime}$ and replacing $A^{\emptyset}$ with $\lim _{\emptyset \neq T \subseteq S^{\prime}} A^{T}$, and let $\mathcal{A}[n]$ be the cartesian $(n-1)$-cube obtained by restricting $\mathcal{A}$ to the complement of $\mathcal{P} S^{\prime}$ 
and replacing $A^{\{n\}}$ with $\lim _{\{n\} \subsetneq T \subseteq S} A^{T}$. Then by induction, the corollary applies to $\mathcal{A}[\emptyset], \mathcal{A}[n]$ and to the square

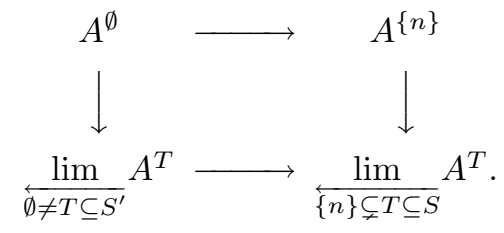

Notice that the conditions in the corollary are unnecessarily restrictive. If for instance $n=3$ we see that demanding that e.g., $A^{\{1\}} \rightarrow A^{\{1,2\}}, A^{\{1,3\}} \rightarrow A^{\{1,2,3\}}$, $A^{\{3\}} \rightarrow A^{\{1,3\}} \times_{A^{\{1,2,3\}}} A^{\{2,3\}}$, and $A^{\emptyset} \rightarrow \lim _{\overleftarrow{\emptyset \neq T}} A^{T}$ are surjective (and the last map has a nilpotent kernel) is enough to conclude that $K(\mathcal{A}) \rightarrow T C(\mathcal{A})$ is cartesian after profinite completion. There are many variants.

\section{Homotopy cartesian squares and $\pi_{0}$}

Theorem 1.1 now follows immediately from Proposition 2.1 and

Proposition 3.1. Let $\mathcal{A}$ be a homotopy cartesian diagram of connective $\mathbf{S}$-algebras

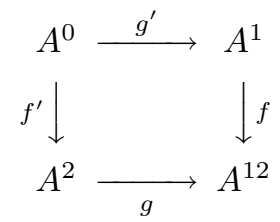

such that $\pi_{0} A^{1} \rightarrow \pi_{0} A^{12}$ is surjective. Then the induced map

$$
h: \pi_{0} A^{0} \rightarrow \pi_{0} A^{1} \times_{\pi_{0}} A^{12} \pi_{0} A^{2}
$$

is a surjection with square-zero kernel.

Proof. First we reduce the proof to the corresponding statement for simplicial rings as found in Lemma 3.2.

Since all $\mathbf{S}$-algebras and the vertical fibers are connective, we may use $\Gamma$-spaces as our model for spectra, and monoids under the smash product of Lydakis [14] as our model for S-algebras. For details, see [6, Chapter II].

Let $H$ be the Eilenberg-Mac Lane construction sending a simplicial ring to a connective $\mathbf{S}$-algebra. The functor $\tilde{\mathbf{Z}}$ which sends a pointed set $X$ to the free abelian group $\tilde{\mathbf{Z}} X=\mathbf{Z}[X] / \mathbf{Z}[*]$ extends to an endofunctor on the category of connective $\mathbf{S}$ algebras. Furthermore, there is a functor $R$ from connective $\mathbf{S}$-algebras to simplicial rings and a natural chain of stable equivalences connecting $\tilde{\mathbf{Z}}$ and $H R$. Proofs may be found in the published version [4, Proposition 3.5] or more directly applicable in [6, Corollary II.2.2.5].

Now, the functor $\tilde{\mathbf{Z}}$ preserves homotopy cartesian diagrams of $\Gamma$-spaces, and so if $\mathcal{A}$ is a homotopy cartesian diagram of connective $\mathbf{S}$-algebras, then $\tilde{\mathbf{Z}} \mathcal{A}$ is a homotopy cartesian diagram of connective $\mathbf{S}$-algebras which is equivalent to $H$ of a homotopy cartesian diagram $R \mathcal{A}$ of simplicial rings. 
Furthermore, the obvious map $\mathcal{A} \rightarrow \tilde{\mathbf{Z}} \mathcal{A}$ is 1-connected (see e.g., [4, Proposition $3.3]$ ), and so we get isomorphisms

$$
\pi_{0} \mathcal{A} \cong \pi_{0} \tilde{\mathbf{Z}} \mathcal{A} \cong \pi_{0} H R \mathcal{A} \cong \pi_{0} R \mathcal{A}
$$

of squares of rings. Lemma 3.2 applied to the homotopy cartesian square $R \mathcal{A}$ of rings, then gives the result.

Lemma 3.2. Let $\mathcal{A}$ be a homotopy cartesian diagram of simplicial rings

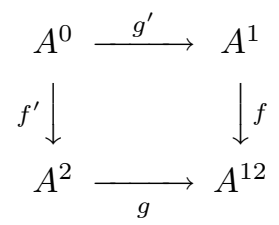

such that $\pi_{0} A^{1} \rightarrow \pi_{0} A^{12}$ is surjective. Then the induced map

$$
h: \pi_{0} A^{0} \longrightarrow \pi_{0} A^{1} \times_{\pi_{0} A^{12}} \pi_{0} A^{2}
$$

is a surjection with square-zero kernel.

Proof. Chasing long exact sequences of homotopy groups yields that $h$ is surjective.

In proving that the kernel of $\pi_{0} A^{0} \rightarrow \pi_{0} A^{1} \times_{\pi_{0} A^{12}} \pi_{0} A^{2}$ is square-zero, the idea is to pick two elements in $\operatorname{ker}(h) \subseteq \pi_{0} A^{0}$ and show, by making an appropriate choice of representatives, that the product of the representatives is homotopic to 0 in $A^{0}$. This implies that the kernel is square-zero. The proof is an exercise in manipulating simplicial homotopies and we refer to [15] for details. For homotopic simplices $x$ and $y$ in a simplicial abelian group $G$, we write $x \sim y$. If $x$ and $y$ happen to be zerosimplices, then being homotopic means that there is a 1-simplex $z$ with $d_{0} z=x$ and $d_{1} z=y$.

We may assume that $A^{1} \rightarrow A^{12}$ is a fibration. According to [17, p. II.3.10], maps of simplicial groups are surjective if and only if they are both fibrations and 0-connected, and so the assumption that $\pi_{0} A^{1} \rightarrow \pi_{0} A^{12}$ is surjective implies that $A^{1} \rightarrow A^{12}$ is a surjection.

Let $\left[u_{0}\right] \in \operatorname{ker}(h)$ be represented by $u_{0} \in A_{0}^{0}$. Then $h\left(\left[u_{0}\right]\right)=\left(\left[f^{\prime} u_{0}\right],\left[g^{\prime} u_{0}\right]\right)=0$ in the pullback, and $f^{\prime}\left(u_{0}\right) \sim 0$ and $g^{\prime}\left(u_{0}\right) \sim 0$ as 0 -simplices in $A^{2}$ and $A^{1}$ respectively. The homotopies are given by 1-simplices $u_{2} \in A_{1}^{2}$ with $d_{0} u_{2}=f^{\prime} u_{0}$ and $d_{1} u_{2}=0$ and $u_{1} \in A_{1}^{1}$ with $d_{0} u_{1}=g^{\prime} u_{0}$ and $d_{1} u_{1}=0$. These simplices correspond to based maps $u_{2}: I \rightarrow A^{2}$ and $u_{1}: I \rightarrow A^{1}$ respectively and by abuse of notation we name the maps after their corresponding simplices. As $u_{0}$ is a 0 -simplex of $A^{0}$ it corresponds to a based map $u_{0}: S^{0} \rightarrow A^{0}$. All this fits into the following diagram of pointed simplicial sets

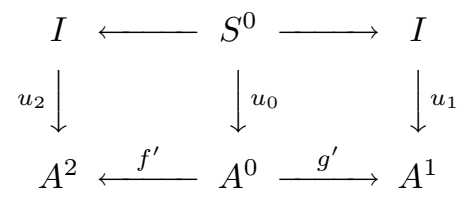

and it represents an element in $\operatorname{ker}(h)$. 
Since $f^{\prime}$ is a fibration of simplicial rings, we may lift $u_{2}: I \rightarrow A^{2}$ to a based map $u: I \rightarrow A^{0}$. It will usually not be compatible with the map $u_{0}: S^{0} \rightarrow A^{0}$, but we do have that $\left[u_{0}\right]=\left[u_{0}-d_{0} u\right]$ and $f^{\prime}\left(u_{0}-d_{0} u\right)=0$, showing that it is enough to consider the situation where $u_{2}=0$, that is, diagrams of the form

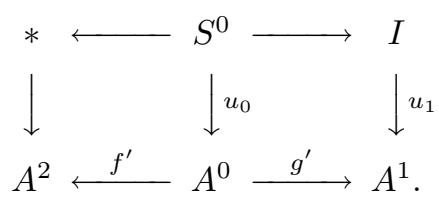

This diagram induces a (based) map $u_{12}: S^{1} \rightarrow A^{12}$, and is our "appropriate choice".

Let $\left[u_{0}\right]$ and $\left[v_{0}\right]$ be any two elements in $\operatorname{ker}(h)$ and pick representatives for them as in diagram (3.1).

Consider the map $s_{0}\left(g^{\prime} u_{0}\right) \cdot v_{1}: I \rightarrow A^{1}$. The dot denotes multiplication in $A^{1}$. The map is a simplicial homotopy from $g^{\prime}\left(u_{0}\right) \cdot g^{\prime}\left(v_{0}\right)$ to 0 since

$$
d_{1}\left(s_{0}\left(g^{\prime} u_{0}\right) \cdot v_{1}\right)=d_{1} s_{0}\left(g^{\prime} u_{0}\right) \cdot d_{1}\left(v_{1}\right)=g^{\prime}\left(u_{0}\right) \cdot 0=0,
$$

and

$$
d_{0}\left(s_{0}\left(g^{\prime} u_{0}\right) \cdot v_{1}\right)=d_{0} s_{0}\left(g^{\prime} u_{0}\right) \cdot d_{0}\left(v_{1}\right)=g^{\prime}\left(u_{0}\right) \cdot g^{\prime}\left(v_{0}\right) .
$$

Because we picked a representative $u_{0} \in \operatorname{ker}\left(f^{\prime}\right)$ we get

$$
f\left(s_{0}\left(g^{\prime} u_{0}\right) \cdot v_{1}\right)=s_{0}\left(f g^{\prime}\left(u_{0}\right)\right) \cdot f\left(v_{1}\right)=s_{0}\left(g f^{\prime}\left(u_{0}\right)\right) \cdot f\left(v_{1}\right)=0 \cdot f\left(v_{1}\right)=0 .
$$

The equations above show that we get a well-defined and based map

$$
\left(0, s_{0}\left(g^{\prime} u_{0}\right) \cdot v_{1}\right): I \rightarrow A^{2} \times_{A^{12}} A^{1},
$$

determining a simplicial homotopy from $\left(0, g^{\prime} u_{0} \cdot g^{\prime} v_{0}\right)$ to 0 . Under the weak equivalence $A^{0} \simeq A^{2} \times A^{12} A^{1}$, the element $\left(0, g^{\prime} u_{0} \cdot g^{\prime} v_{0}\right)$ corresponds to the product $u_{0} \cdot v_{0}$, showing that it is homotopic to 0 .

Example 3.3. An example of the situation in Lemma 3.2 where the kernel of $h$ is nontrivial may be helpful. Consider the diagram of simplicial rings

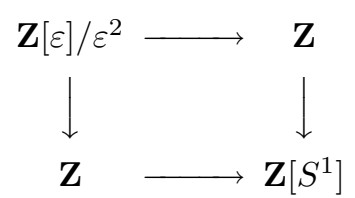

in which both maps to $\mathbf{Z}$ are projections and both maps from $\mathbf{Z}$ are inclusion into the simplicial ring $\mathbf{Z}\left[S^{1}\right]$. All rings in the diagram but $\mathbf{Z}\left[S^{1}\right]$ are discrete. The diagram is homotopy cartesian which is easily checked as the zeroth homotopy groups are the only nontrivial homotopy groups of the fibers. In this case

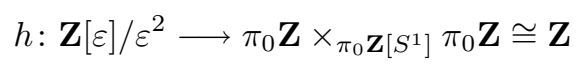

is the projection with square-zero kernel $\mathbf{Z}\langle\varepsilon\rangle$, the infinite cyclic group generated by $\varepsilon$.

The connectivity hypothesis on $f^{1}$ is annoying in that it makes it difficult to state minimal hypotheses for good multirelative versions. As a crude corollary of the main result one has the following: 
Corollary 3.4. Let $\mathcal{A}$ be a homotopy cartesian $S$-cube of connective $\mathbf{S}$-algebras such that for all $U \subseteq S$ the canonical map

$$
p^{U}: A^{U} \rightarrow \underset{U \subsetneq \operatorname{holim}}{\operatorname{LfS}} A^{T}
$$

is 0-connected. Then the $(|S|+1)$-cube

$$
K(\mathcal{A}) \rightarrow T C(\mathcal{A})
$$

induced by the cyclotomic trace is homotopy cartesian after profinite completion.

Note that $p^{\emptyset}$ is an equivalence (and thus 0-connected) because $\mathcal{A}$ is assumed to be homotopy cartesian. When $U=S$, the homotopy limit is taken over the empty set and $p^{S}: A^{S} \rightarrow *$ is clearly 0 -connected.

Proof of Corollary 3.4. The proof of this corollary is exactly as the proof of Corollary 2.2 , except that you remove $\pi_{0}$ (and replace the limits by homotopy limits or replace the cube with a fiber cube so that limits and homotopy limits agree up to stable equivalence).

Remark 3.5. Corollary 3.4 is not optimal. For instance if $n=3$, it would also suffice that the maps $A^{\emptyset} \rightarrow A^{\{3\}}, A^{\{1\}} \rightarrow A^{\{1,2\}}$ and $A^{\{2,3\}} \rightarrow A^{\{1,2,3\}}$ were 0 -connected (in addition to homotopy cartesianness of the cube). Note that this condition is actually not contained in the one given in the corollary, but is one of the many variants possible. We spell out this example.

Let $F$ be the profinite completion of the fiber of the cyclotomic trace. We may assume all maps are fibrations. Then $F$ applied to the squares

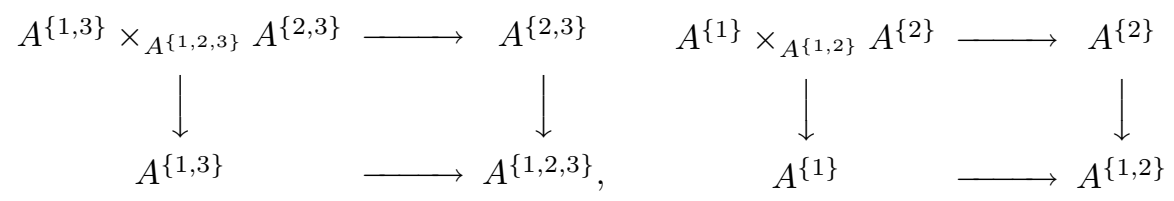

give homotopy cartesian squares.

Consider the square

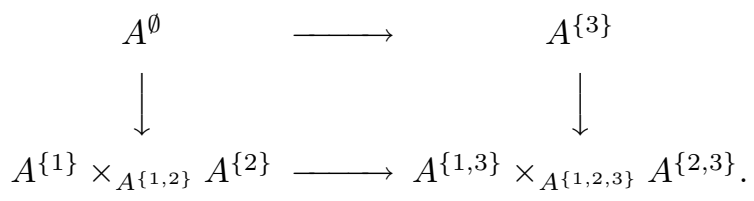

This square is homotopy cartesian since the entire cube is, and by assumption the top map is 0 -connected. Since everything is connective it follows that the bottom map is 0 -connected too, and so the main theorem applies again to show that $F$ applied to this square is homotopy cartesian. Collecting the pieces we get that $F$ applied to the cube is homotopy cartesian.

Theorem 1.1 implies that for $n=2$, we only need $f^{1}$ (or $f^{2}$ ) to be 0 -connected, but the condition in 3.4 requires both to be 0 -connected; this shows again that the statement of 3.4 is not optimal. 


\section{References}

[1] H. Bass, Algebraic K-Theory, W. A. Benjamin, Inc., New York, 1968.

[2] M. Bökstedt, W. C. Hsiang, and I. Madsen, The cyclotomic trace and algebraic K-theory of spaces, Invent. Math. 111 (1993), 465-539.

[3] G. Cortiñas, The obstruction to excision in $K$-theory and in cyclic homology, Invent. Math. 164 (2006), 143-173.

[4] B. I. Dundas, Relative $K$-theory and topological cyclic homology, Acta Math. 179 (1997), 223-242.

[5] B. I. Dundas, The cyclotomic trace for S-algebras, J. London Math. Soc. 70 (2004), 659-677.

[6] B. I. Dundas, T. Goodwillie, and R. McCarthy, The local structure of algebraic K-theory, http://www.math.ntnu.no/ dundas/indexeng.html, preprint.

[7] T. Geisser and L. Hesselholt, Bi-relative algebraic $K$-theory and topological cyclic homology, Invent. Math. 166 (2006), 359-395.

[8] S. Geller, L. Reid, and C. Weibel, The cyclic homology and $K$-theory of curves, Bull. Amer. Math. Soc. 15 (1986), 205-208.

[9] S. Geller, L. Reid, and C. Weibel, The cyclic homology and $K$-theory of curves, J. Reine Angew. Math. 393 (1989), 39-90.

[10] S. Geller and C. Weibel, $K(A, B, I)$. II, K-Theory 2 (1989), 753-760.

[11] S. C. Geller and C. A. Weibel, $K_{1}(A, B, I)$, J. Reine Angew. Math. 342 (1983), 12-34.

[12] T. G. Goodwillie, Notes on the cyclotomic trace, Lecture notes for a series of seminar talks at MSRI, spring 1990, December 1991.

[13] J.-L. Loday, Cyclic Homology, Grundlehren Math. Wiss. 301 [Fundamental Principles of Mathematical Sciences], Appendix E by M. O. Ronco, Chapter 13 by the author in collaboration with T. Pirashvili, Springer-Verlag, New York, 1998.

[14] M. Lydakis, Smash products and $\Gamma$-spaces, Math. Proc. Cambridge Philos. Soc 126 (1999), 311-328.

[15] J. P. May, Simplicial Objects in Algebraic Topology, Reprint of the 1967 original, Chicago Lectures in Mathematics, University of Chicago Press, Chicago, IL, 1992.

[16] R. McCarthy, Relative algebraic $K$-theory and topological cyclic homology, Acta Math. 179 (1997), 197-222.

[17] D. G. Quillen, Homotopical Algebra, Lecture Notes in Math. 43, SpringerVerlag, New York, 1967.

[18] A. A. Suslin and M. Wodzicki, Excision in algebraic K-theory, Ann. of Math. 136 (1992), 51-122.

[19] R. G. Swan, Excision in algebraic K-theory, J. Pure Appl. Algebra 1 (1971), 221-252. 
[20] R. W. Thomason and T. Trobaugh, Higher algebraic $K$-theory of schemes and of derived categories, in The Grothendieck Festschrift, Vol. III, Progr. Math. 88, 247-435, Birkhäuser Boston, Boston, MA, 1990.

Bjørn Ian Dundas dundas@math.uib.no

Department of Mathematics, University of Bergen, Norway

Harald Øyen Kittang Harald-Oyen.Kittang@iu.hio.no

Faculty of Engineering, Oslo University College, Norway 